Teknokultura. Revista de Cultura Digital y Movimientos Sociales

ISSNe: $1549-2230$

https://dx.doi.org/10.5209/TEKN.69303

\title{
Conversando con cajas negras; sobre la aparición de los interfaces conversacionales ${ }^{1}$
}

\author{
Raúl Tabarés Gutiérrez ${ }^{2}$
}

Recibido: 12 de mayo de 2020 / Aceptado: 8 de julio de 2020 Open peer reviews

Resumen. En los últimos años hemos asistido a la aparición casi simultánea de diferentes interfaces conversacionales impulsadas por diversos representantes del capitalismo digital. Dichos interfaces han sido implementados en una variedad de dispositivos y objetos con la finalidad de facilitar la interacción entre humanos y computadoras. Sin embargo, dichas interfaces plantean nuevas problemáticas que están íntimamente asociadas con los modelos de negocio que guían su desarrollo tecnológico, a través de la denominada como "economía de las plataformas". En el presente texto se hace una revisión de la literatura en torno a dichas plataformas que impulsan el capitalismo digital, prestando especial atención a la aparición de los nuevos interfaces conversacionales. Asimismo, se presta especial atención al proyecto CALO, financiado por la DARPA, y que constituye el germen de muchas de estas innovaciones. En el artículo se argumenta que la introducción de este tipo de interfaces conversacionales facilita la introducción de nuevas desigualdades y amenazas en el espacio online para las comunidades más minoritarias y menos empoderadas por las tecnologías digitales.

Palabras clave: automatización; asistentes virtuales; capitalismo digital; economía de las plataformas; IA.

\section{[en] Speaking with black boxes; about the rising of conversational interfaces}

\begin{abstract}
In recent years we have observed how almost simultaneously different conversational interfaces have been pushed forward by various players of digital capitalism. These interfaces have been implemented in a variety of devices and artefacts for facilitating interaction between humans and computers. However, these interfaces pose new problems that are intimately associated with the business models that guide their technological development, through the so-called "platform economy" paradigm. In the text, a literature review of platforms that fuel digital capitalism is presented, paying special attention to the appearance of new conversational interfaces. In addition, special attention is paid to the CALO project, funded by DARPA, and commonly seen as a backbone of these innovations. The paper argues that these conversational interfaces facilitates the introduction of new inequalities in online space, which threaten marginalized and less-empowered communities with regards to digital technologies.
\end{abstract}

Keywords: AI; automation; digital capitalism; platform economy; virtual assistants.

Sumario. 1. Introducción. 2. La economía de las plataformas y la emergencia de la IA. 3. Hablando con la Web. 4. Imitando la voz humana. 5. La voz como moneda digital. 6. Nuevas interfaces para nuevas desigualdades. 7. Conclusiones y futuras direcciones. 8. Referencias.

Cómo citar: Tabarés Gutiérrez, R. (2020). Conversando con cajas negras; sobre la aparición de los interfaces conversacionales. Teknokultura. Revista de Cultura Digital y Movimientos Sociales, 17(2), 179-186.

\section{Introducción}

Durante los últimos años hemos visto cómo el desarrollo de nuevas técnicas de inteligencia artificial (IA) y aprendizaje automático, del inglés Machine Learning (AA) ha permitido un gran avance en el desarrollo de interfaces conversacionales (IC) en forma de asistentes virtuales, chatbots y robots sociales, entre otros. Estas innovaciones han surgido de la mano de diversas plataformas digitales que copan los primeros puestos en capitalización bursátil y se han posicionado como claros exponentes del nuevo capitalismo digital basado en la explotación económica de los datos generados por sus usuarios. Sin embargo, la adopción por parte de la ciudadanía de este nuevo tipo de interfaces basadas en la voz humana plantea una serie de problemáticas, alineadas con los propios modelos de negocio digitales y la falta de inclusividad, equidad y responsabilidad que se plantea en el desarrollo de este tipo de tecnologías.

Así, el presente texto trata de aportar una mirada crítica a estas innovaciones, analizando en detalle el surgimiento de las IC durante los últimos años y delimitando

\footnotetext{
Este trabajo ha sido posible gracias al proyecto PLUS (Platform Labour in Urban Spaces: Fairness, Welfare, Development), financiado por la Comisión Europea a través del programa Horizonte 2020 y con número de contrato 822638.

2 TECNALIA Ventures, Basque Research and Technology Alliance (BRTA) (España).

E-mail: raul.tabares@tecnalia.com
} 
cuáles son sus implicaciones sociales a largo plazo. Para ello, este estudio presenta los resultados de una revisión de la bibliografía asociada al desarrollo de las IC y de las plataformas que las impulsan, prestando especial atención al proyecto CALO como germen de todas ellas, y aportando posibles direcciones futuras para continuar la investigación en torno a las IC y sus implicaciones socio-éticas. El texto en cuestión se estructura en torno a una revisión de la economía de las plataformas y la emergencia de la IA (2), el desarrollo de las IC (3), sus precedentes históricos (4), la crítica a sus modelos de negocio (5), el desarrollo de desigualdades digitales asociadas a las mismas (6) y unas conclusiones (7) que recogen futuras direcciones de la investigación en este campo.

\section{La economía de las plataformas y la emergencia de la IA}

Durante los últimos años, hemos sido testigos de cómo diferentes plataformas digitales se han convertido en actores comunes de nuestra vida cotidiana. De esta manera Facebook, YouTube, Netflix, Uber o Airbnb se han convertido en compañeras habituales para mantener el contacto con nuestros amigos, ver películas de forma online o alquilar un alojamiento durante nuestras vacaciones. Todos estos ejemplos son representantes de un nuevo tipo de economía que está surgiendo al albor del desarrollo de Internet y la Web, en forma de plataformas digitales (M. L. Gray \& Suri, 2019; Kenney \& Zysman, 2016; Srnicek, 2017; van Dijck, Poell, \& Waal, 2018). Dichas plataformas comparten ciertas características que combinan tecnología, marketing, procesos y aspectos organizativos innovadores, así como otras connotaciones políticas y mediáticas que no estaban presentes en anteriores formas de organización empresarial. Estas plataformas digitales parecen ser una moda pasajera, pero al mismo tiempo, una tendencia general en el tipo de economía que promueve el capitalismo digital (Gillespie, 2010). Por ello, no es fácil entender las plataformas digitales en su conjunto, ya que presentan las características de un mercado horizontal, mientras que por otro lado mantienen la jerarquía típica de las empresas privadas (Sundararajan, 2016). Una de las definiciones más difundidas sobre las plataformas digitales categoriza dichos artefactos como infraestructuras basadas en la recolección de datos y su clasificación, donde estos datos son producidos por la misma interacción que los usuarios tienen con la plataforma en sí misma (Srnicek, 2017). Por el contrario, otras definiciones hacen énfasis en el término 'economía de las plataformas' y lo enfatizan como: un término que congrega un creciente número de actividades digitalmente habilitadas en la economía, la política y la interacción social (Kenney \& Zysman, 2016, p. 62).

Dichas plataformas digitales se han beneficiado en gran medida de las connotaciones positivas iniciales asociadas a la 'economía colaborativa' (Sundararajan, 2016), el 'consumo colaborativo' (Botsman \& Rogers, 2011) o la 'colaboración abierta' (Stefano, 2016), pero también de la libre disposición de bienes comunes digitales, en forma de contenidos generados por el usuario (CGU) (Ritzer \& Jurgenson, 2010), facilitado a través de nuevas infraestructuras tecnológicas desarrolladas ad hoc (Gutiérrez, 2018). El término plataforma ha sido promovido por varios representantes de este nuevo tipo de economía que promueve el capitalismo digital, bajo la influencia de la 'ideología californiana' (Barbrook \& Cameron, 1996), con el propósito de establecer un ecosistema neutral donde los usuarios puedan ser tratados de forma igualitaria (Turner, 2006). Al mismo tiempo, estos ecosistemas virtuales se han posicionado en la sociedad como intermediarios culturales, buscando en todo momento modelos de negocio sostenibles y auto promoviéndose como defensores de la libertad de expresión (Gillespie, 2010). Sin embargo, no hay que olvidar que dichas plataformas dependen totalmente de la contribución de sus usuarios y la digitalización de las actividades humanas a la hora de la generación de valor comercial (M. L. Gray \& Suri, 2019; Kenney \& Zysman, 2016; Scholz, 2012; Terranova, 2000; van Dijck et al., 2018).

La popularización de dichos servicios durante el surgimiento de la Web 2.0 (O'Reilly, 2005), así como la irrupción de las redes sociales han contribuido de manera decisiva a la generación de enormes bases de datos a través de plataformas CGU (van Dijck, 2013). Además, la popularización y adopción masiva de los dispositivos móviles por parte de la ciudadanía (Vogelstein, 2013) también ha permitido a los impulsores de este capitalismo digital acceder a diferentes tipos de datos relacionados con sus usuarios, rutinas, hábitos y comportamientos. Datos que no son introducidos por el usuario, sino recolectados por los dispositivos a través de la interacción de estos con otros dispositivos y con el medio (a través de técnicas como la geolocalización o a través de tecnologías como RFID o bluetooth). Así se produce una generación de bienes digitales, en forma de datos, metadatos o datos agregados, en los que el usuario de las plataformas participa de su recolección y agregación, ya sea de forma activa y/o pasiva, al aceptar los famosos términos de referencia que cada una de estas empresas obliga a aprobar previo uso del servicio.

Esto ha conllevado que diversos términos hayan aparecido durante los últimos años para clasificar este tipo de dinámicas que se producen en el capitalismo digital tales como 'capitalismo de vigilancia' (Zuboff, 2019), 'trabajo digital' (Scholz, 2012), 'trabajo gratis' (Terranova, 2000) o más recientemente 'trabajo fantasma' (M. L. Gray \& Suri, 2019). Todos los anteriores conceptos reflejan la centralidad adquirida por las plataformas digitales en la economía y la sociedad, además de los nuevos modos de extracción de la plusvalía que se ejercen no solamente en los trabajadores remunerados (tanto internos como externos a la organización), sino también en los consumidores y usuarios de los servicios ofrecidos a través de dichas plataformas.

Además, los recortes y precarización que han asolado a multitud de sectores de actividad tras la gran recesión del 2008 han ahondado en la progresiva liberalización del mercado laboral iniciada en los años '80, propician- 
do que formas alternativas de trabajo sean cada vez más comunes (Scholz, 2017; Srnicek, 2017). Esto unido a la digitalización creciente de la socialización y el efecto de descentralización que la cultura asociada a Internet provoca en la sociedad (van Dijck, 2013), han acelerado esta transición hacia los servicios digitales ofertados por estas nuevas formas de organización empresarial. En este sentido Zenney y Zysman reflejan su preocupación ante tal tendencia general:

Si la revolución industrial se organizó en torno a la factoría, los cambios que estamos viendo giran en torno a las plataformas digitales, vagamente definidas. De hecho, estamos en una reorganización de nuestra economía en la que los propietarios de las plataformas están desarrollando un poder, aparentemente, que puede ser aún más formidable que el que tuvieron los propietarios de las fábricas a comienzos de la revolución industrial (Kenney \& Zysman, 2016, p. 62).

Desde sus inicios, la evolución de estas plataformas se ha centrado principalmente en el desarrollo de herramientas de Big Data (C. Gray, 2014), conllevando una reducción drástica de los costes de recopilación y almacenamiento de datos, pero propiciando también el desarrollo de técnicas de procesamiento mucho más avanzadas para grandes conjuntos de datos (O'Neill, 2017). Al mismo tiempo, las plataformas digitales han ido incluyendo en sus diversos componentes (código, sistemas, dispositivos protocolos, etc.) un buen número de algoritmos que procesan, organizan y categorizan grandes conjuntos de datos. Estos algoritmos se caracterizan por disponer de una opacidad significativa, ya que se necesita de unas habilidades específicas para comprender su funcionamiento interno, además de que resulta difícil delimitar en qué parte exacta de la plataforma se encuentran y cuál es su forma concreta. Además, habitualmente se encuentran protegidos por leyes de propiedad intelectual lo cual dificulta aún más su discernimiento, convirtiéndolos en formidables cajas negras (Dourish, 2016; Pasquale, 2015).

Por ello, los algoritmos se convierten en activos muy valiosos en el capitalismo digital, no simplemente a la hora de capturar, recopilar, almacenar y procesar datos, sino como elementos activos que pueden aprender por sí mismos, proporcionando nuevas rutas para la innovación en forma de oportunidades y servicios, contribuyendo a su vez, a fortalecer la competitividad de las plataformas digitales y su posicionamiento en el mercado (O’Neill, 2017). Asimismo, el tratamiento de datos no es algo radicalmente nuevo en lo que respecta a las estrategias empresariales, ya que ha sido algo muy utilizado por los medios de masas, que han experimentado con técnicas como las audiencias de prueba, las calificaciones (ratings) y otros (Hallinan \& Striphas, 2014). Sin embargo, los grandes avances en IA, tales como el AA, el aprendizaje profundo, del inglés Deep Learning (AP) o el procesamiento de lenguaje natural, del inglés Natural Language Processing (PLN) han conllevado toda una serie de innovaciones sin precedentes como los vehículos autónomos, el trading algorítmico (Pasquale,
2015), el mantenimiento predictivo en la Industria 4.0 (Gutiérrez \& Ezponda, 2019) y los asistentes virtuales (Guzman, 2018), que han impulsado notablemente las posibilidades de las ICs (Geller, 2012).

\section{Hablando con la Web}

Las IC se están abriendo paso en diversos ámbitos de la cotidianidad y sobremanera en forma de asistentes virtuales (Guzman, 2018), robots sociales, chatbots, altavoces inteligentes y otro tipo de innovaciones similares. La orientación hacia el usuario de estos 'lazarillos digitales' tratan de proveer asistencia en la intimidad del hogar (aunque no exclusivamente), de la automatización de tareas rutinarias a través de la conexión y mediación con otros servicios digitales (Phan, 2019). Dichas tecnologías se difunden progresivamente en la sociedad con la promesa de facilitar y simplificar la comunicación entre humanos y máquinas. Así, la mayoría de los representantes de la denominada economía de las plataformas están promocionando sus propios interfaces basados en la voz humana con nombres tan conocidos como Alexa, Cortana, Google Now o Siri, entre otros. Dicho fenómeno no es exclusivo de occidente, ya que la misma lógica está siendo seguida por los representantes de este paradigma en China, de la mano de compañías como Alibaba, Baidu y Tencent (Knight, 2016a).

Este impulso a las IC es posible por los grandes avances realizados en el campo del PLN recientemente, gracias a la disponibilidad masiva de datos provenientes de servicios 2.0 (van Dijck, 2013), el coste cada vez menor de computadoras avanzadas y unidades de procesamiento gráfico, del inglés Graphic Process Unit (GPU), además de las nuevas posibilidades que ha propiciado la computación en la nube (van Dijck et al., 2018). Así, las IC generan nuevas expectativas por parte de diferentes sectores y en lo que se refiere a nuevos modos de interacción entre humanos y máquinas.

Cuadro. 1. Icono habilitado en dispositivos de Apple para ejecutar el asistente virtual Siri. Fuente: Wikimedia.

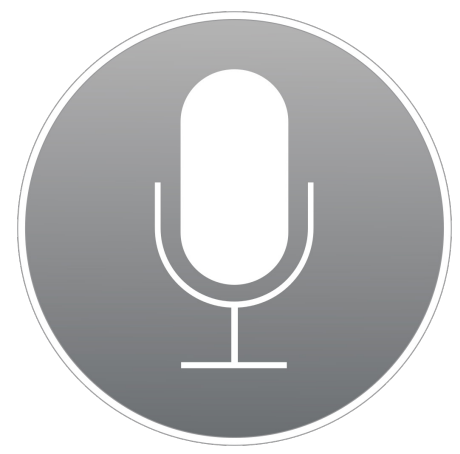

No hay que olvidar que este tipo de interfaces han estado muy presentes en la literatura de ciencia-ficción (Allen et al., 2001), el cine y las series de televisión, con ejemplos destacados como 2001: Una odisea del espacio o Ella. Comúnmente se ha argumentado que el habla es la tecnología que llevará Internet definitivamente a toda 
la población (Lai, 2000), ya que el lenguaje hablado es el medio más natural, eficiente, flexible y barato de comunicación entre humanos (Zue \& Glass, 2000).

No en vano, estos artefactos fueron identificados como una de las 10 tecnologías innovadoras para 2016 por el MIT (Knight, 2016a) y las expectativas en torno al desarrollo de estas tecnologías han crecido constantemente durante los últimos años. Dichas innovaciones parecen enmarcarse en la lógica de nuevas interfaces que impulsa el capitalismo digital desde el surgimiento de la etapa conocida como Web 2.0, y donde se rompe por primera vez la exclusividad del PC como puerta de entrada a la Web (O'Reilly, 2005). El primero de los dispositivos que parece romper masivamente esa dualidad es el iPod, ya que permite la transferencia de archivos a un dispositivo móvil, pero la posterior irrupción de teléfonos inteligentes y tablets (Vogelstein, 2013), continuará y aumentará esa fragmentación de dispositivos y accesos a la Web (Gutiérrez, 2018). Estas nuevas puertas de entrada a la Web no sólo modificarán de forma radical la forma en que el usuario interactúa con la Web, promoviendo el uso de controles táctiles entre otros, sino que también propulsarán la involucración de un mayor número de usuarios previamente no familiarizados con el ciberespacio (Gutiérrez, 2016). Además, diversos modelos de negocio propulsados por las diversas plataformas digitales que irrumpen con fuerza durante esta época empiezan a ser contrastados y asentados (Scholz, 2012; Terranova, 2000; van Dijck, 2013).

Es poco después de este periodo, cuando en 2011 Apple decide presentar en sociedad a 'Siri', su asistente virtual, integrado en la versión $4 \mathrm{~S}$ de su modelo de teléfono inteligente iPhone (ver cuadro 1). Siri es un asistente virtual que es propiedad de la compañía norteamericana y forma parte de sus diferentes sistemas operativos y dispositivos (Phan, 2017). El asistente interactúa con el usuario que utiliza el dispositivo a través de una interfaz basada en el habla para responder preguntas, hacer recomendaciones o realizar acciones en otros servicios digitales a los que es capaz de conectarse bajo petición, proporcionando resultados de manera individualizada y orientada al usuario con el que interactúa (Guzman, 2017). La presentación del asistente se produjo después de la adquisición por parte de Apple de la compañía que contaba con la tecnología que hacía posible este asistente virtual y que provenía del Centro Internacional de Inteligencia Artificial SRI (Roush, 2010). Siri era una de las ramificaciones de un colosal proyecto de I+D financiado por la DARPA (Defensive Advanced Research Projects Agency), bajo su programa PAL (Personalized Assistant that Learns) y cuyo título era CALO (Cognitive Assistant that Learns and Organizes) (Artificial Intelligence Center, s.f.; Geller, 2012). Dicha iniciativa aglutinó a 300 investigadores de gran calado de la industria y la academia durante 5 años (2003-2008), con el objetivo de: crear sistemas de software cognitivo, es decir, sistemas que puedan razonar, aprender de la experiencia, que se les diga qué hacer, explicar lo que están haciendo, reflexionar sobre su experiencia y responder con firmeza para sorprender (Artificial Intelligence Center, s.f.).

El nombre de CALO se inspiraba en la palabra latina ‘calonis' que significa 'sirviente del soldado' y además de Siri, hubo varios resultados de la investigación reseñables, tales como 'Social Kinetics' (una aplicación social capaz de aprender estrategias personalizadas de tratamiento e intervención en pacientes con enfermedades crónicas), 'Tempo AI' (un calendario inteligente) o 'Desti' (una guía de viaje personalizada). Uno de los grandes avances de este proyecto residió en demostrar que una máquina puede aprender en tiempo real y que diferentes y complejas disciplinas de la IA pueden cooperar juntas. Al mismo tiempo, también abrió las puertas a nuevas posibilidades dentro del AA, ya que cada componente del proyecto aprendió 'in vivo' de una dieta incontrolada de información, en vez de un conjunto establecido de datos (Bosker, 2013).

El principal objetivo del proyecto CALO se enmarcaba en el desarrollo de una herramienta que fuera capaz de gestionar activamente una agenda personal, organizando diferentes reuniones al mismo tiempo, completando acciones en nombre del usuario, así como otro tipo de tareas relacionadas. Dicho de otro modo, sus creadores querían desarrollar 'una máquina de hacer cosas', bajo un nuevo enfoque que permitiera la automatización en las consultas a los motores de búsqueda: con el propósito de crear algo que permitiera a las personas mantener conversaciones con Internet (Bosker, 2013). Este innovador enfoque fue crítico, ya que permitió a Siri rastrear el contenido de una pregunta en busca de acciones potenciales y posteriormente, seleccionar la acción más probable basándose en diversas preferencias del usuario, tales como su localización, la fecha y hora y/o requerir más información (Guzman, 2017). Un logro facilitado en buena medida por la abundancia de datos provenientes de dispositivos móviles y plataformas digitales, tal y como reconocía alguno de los investigadores involucrados en la iniciativa: no sería posible hacer algo como Siri hace 10 o 15 años porque no se podría obtener suficientes datos para entrenar al sistema (Geller, 2012, p. 15).

Cuadro. 2. Gama de altavoces Echo que incluyen el asistente virtual Alexa. Fuente: Wikimedia

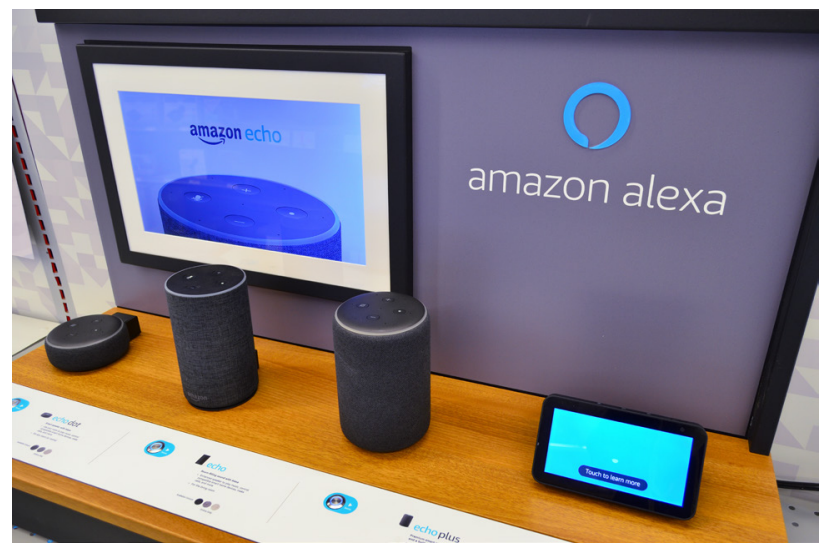

Otras compañías pujantes del capitalismo digital, tales como Amazon, Google o Microsoft, no dejaron pasar la oportunidad de desarrollar sus propios asistentes virtuales y así en los últimos años han ido surgiendo Alexa, Goole Now o Cortana, entre otros (Bell, 2017; Guzman, 2018). 
Dichos asistentes también usan una IC y son desplegados en los diversos servicios que operan estas compañías tales como sistemas operativos, motores de búsqueda para la Web o aplicaciones ofimáticas, entre otros. El caso más diferente de uso de estos asistentes virtuales sea el de Alexa por parte de Amazon, el cuál fue concebido de manera íntimamente asociada a su altavoz inteligente 'Echo' (ver cuadro 2). Esta asociación ha facilitado la interacción con otros dispositivos inteligentes en el hogar y por ende, la automatización de tareas rutinarias a través de otros servicios digitales (Phan, 2019).

\section{Imitando la voz humana}

Varios de los asistentes digitales citados en el presente texto tienen precedentes históricos como ELIZA (1966), PARRY (1972) o ALICE (1995). Todos estos programas informáticos fueron concebidos para participar en concursos como el 'Premio Loebner', que han sido fuertemente influenciados por la obra de Turing, uno de los fundadores de la IA y especialmente por su famoso artículo publicado en 1950 titulado 'Computing Machinery and Intelligence'. En dicho texto se proponía lo que posteriormente se ha conocido como 'El Test de Turing' y dónde el autor se inquiría asimismo si una máquina puede comportarse de manera idéntica a una mente humana (Turing, 1950). Con esta prueba Turing no se preguntaba si una máquina era capaz de pensar, sino solamente si podía hacerse pasarse por humano en una conversación ${ }^{3}$. El trabajo de Turing fue muy influyente, al mismo tiempo que criticado, ya que la prueba solamente se centraba en verificar si la computadora se comportaba como un ser humano o no. No en si la máquina es capaz de mostrar inteligencia. A pesar de estas críticas, no cabe duda de que Turing realizó un primer paso conceptual que influiría sobremanera en el desarrollo de la IA, las IC y el PLN.

Es en este último campo donde la informática, la IA y la lingüística computacional se entremezclan, con el objetivo de mejorar las interacciones entre las computadoras y los diversos lenguajes humanos para procesar grandes corpus lingüísticos. Con los recientes avances a los que estamos asistiendo en materia de AA, como el AP y el reconocimiento de voz automatizado, gracias a la abundancia de grandes cantidades de datos para entrenar computadoras (Geller, 2012; M. L. Gray \& Suri, 2019; van Dijck, 2013) estamos viendo como estas IC tan populares en la literatura de ciencia-ficción están dejando de ser ficción. Al mismo tiempo, nuevas competiciones similares al Premio Loebner, como el $\mathrm{Wi}$ nograd Schema Challenge han sido publicitadas en los últimos años, con el fin de proponer nuevos retos para el desarrollo de este tipo de tecnologías, tales como la comprensión de oraciones que son ambiguas, y que al mismo tiempo son simples de diseccionar para los humanos (Knight, 2016b).

La prueba partía de la premisa de que no todos los comportamientos humanos son inteligentes, al mismo tiempo que muchos comportamientos de computadoras pueden ser inteligentes. El ser humano comete errores continuamente y las máquinas deben incorporar este tipo de errores para poder parecer humanas.

\section{Minando la voz humana}

Como hemos visto a lo largo del texto, las IC han sido posicionadas por diversas plataformas digitales como un gran salto hacia adelante en términos de interfaz humano-computadora. Dichas innovaciones han sido promovidas en torno al valor diferencial que aportan al usuario a la hora de interconectar diversos dispositivos, aplicaciones y sistemas, facilitando su interconexión e introduciendo la automatización a través de los controles de voz para facilitar diversas tareas. Podríamos definir este tipo de innovaciones como todo punto de comunicación entre el usuario y el computador que tiene la capacidad de imitar a un humano a través de una conversación mediada por una plataforma de comunicación tecnológica, $y$, por lo tanto, puede proveer de una experiencia cuasi humana al usuario con el que interactúa. Dicho con otras palabras, podríamos categorizar a las IC como innovaciones que permiten interactuar al usuario con un computador de forma textual y/o verbal y cuyas interacciones son digitalizadas para ser posteriormente almacenadas, analizadas y procesadas a través de diversas técnicas de IA.

Como hemos visto, los asistentes virtuales han surgido con fuerza dentro de las IC, pero otras innovaciones como chatbots o robots sociales, al igual que formas más complejas como hologramas en $3 \mathrm{D}$, dispositivos hápticos o coches autónomos puede que implementen este tipo de interfaces en un futuro cercano. Más si cabe con los crecientes procesos de digitalización que se están produciendo en diversos sectores y el impulso que recibirán los interfaces no táctiles a raíz de la crisis del COVID-19. Entre las ventajas que se asocian a estas innovaciones suele argumentar que los comandos, menús, iconos y demás podrían ser reemplazados por comandos de voz o palabras clave, simplificando la interacción con el usuario. También se ha argumentado que las IC pueden favorecer la inclusividad de personas que padecen algún tipo de discapacidad visual o de otro tipo. Quizás, la mayor aspiración radique en que estas interfaces aspiran a proporcionar una forma de comunicación 'más natural' entre humanos y máquinas, tratando de evitar las fricciones recurrentes que muchos usuarios sufren en su día a día cuando se conectan a la Web. Aquí las IC podrían posicionarse como un elemento que pudiera favorecer el acceso a los diversos servicios que proporcionan Internet y la Web para los usuarios que todavía experimentan dificultades a la hora de acceder a estas redes.

Sin embargo, esta transición no está libre de coste para el usuario. El desarrollo de técnicas de IA alrededor de las IC obedece en su mayor parte a intereses económicos de los grandes representantes de la 'economía de las plataformas', en su búsqueda de nuevos servicios asociados a la recolección, almacenaje y tratamiento de datos de usuarios. El leitmotiv de todas estas innovaciones centradas en sus usuarios obedece a la lógica que persigue la rentabilidad económica a corto plazo de la I+D (M. L. Gray \& Suri, 2019; Scholz, 2012; van Dijck et al., 2018). En este sentido, la voz humana parece ser la nueva frontera que conquistar por parte de este 
tipo de modelos de negocio que impulsa el capitalismo digital. Una nueva minería digital que pretende excavar y extraer el valor asociado a la voz y al habla, de cara al desarrollo y popularización de las IC, gracias a la omnipresencia de las plataformas digitales en nuestra sociedad y la creciente digitalización de las interacciones sociales. Es por ello que las diversas innovaciones que han posicionado estas plataformas digitales durante los últimos años, buscan extender en todo momento su rango de acción (M. L. Gray \& Suri, 2019; van Dijck, 2013). Así, nos encontramos ante un ecosistema tecnosocial, extendido por diversas plataformas, dispositivos, protocolos, algoritmos e interfaces que buscan en todo momento digitalizar y valorizar las interacciones de sus usuarios con el medio. Un entramado que además ha sido desplegado por un número limitado de empresas norteamericanas y afincadas en el famoso 'Valle del Silicio' y cuya congregación de poder, recursos e influencia no parece tener parangón con otros episodios históricos de la industrialización (Kenney \& Zysman, 2016).

Las IC nos brindan más facilidades a la hora de interactuar en el plano digital, pero también de una mayor opacidad a la hora de conocer qué ocurre detrás del artefacto. Más dificultades para entender cómo funciona el sistema, los dispositivos y sus procesos, así como para entender la lógica capitalista que impulsa su diseño tecnológico y que está inherentemente incrustada en su diseño. Así, vemos cómo el dispositivo obedece a las palabras del usuario, al mismo tiempo que se introducen más barreras para entender el sistema. Por ello, y pese a que el usuario sienta que dispone de más facilidades a la hora de acceder al espacio cibernético, lo cierto es que su vulnerabilidad crece. De esta manera, la inhabilidad para conocer y discernir las interacciones que se producen a través del plano online y que son mediadas por nuevas interfaces más opacos, hacen que fenómenos como la desinformación, las noticias falsas, el phishing o técnicas avanzadas de la mercadotecnia como la micro-focalización puedan ser operadas con mayor facilidad y frecuencia, ante nuestra creciente incapacidad para conocer el medio en el que nos desenvolvemos.

\section{Nuevas interfaces para nuevas desigualdades}

Como hemos visto a lo largo del texto, el posicionamiento de las plataformas digitales como intermediarios culturales durante los últimos años ha jugado un papel clave en la centralidad que ocupan ahora mismo en la sociedad y en diversos ámbitos como el transporte, la educación o la búsqueda de información (Gillespie, 2010; van Dijck et al., 2018). Estos esfuerzos por adoptar un posicionamiento neutral radica en ideologías ciber-libertarias que heredan valores que se impulsaron en los años ' 70 en la costa oeste norteamericana y que en buena medida influyeron a toda una generación de emprendedores en Internet (Barbrook \& Cameron, 1996; Turner, 2006). Sin embargo, los recurrentes problemas que dichas plataformas enfrentan en torno a la moderación de contenidos, su falta de regulación, sus sesgos ideológicos, la priorización de sus propios intereses comerciales dentro de dichos entornos y la predisposición técnica a la viralización de contenidos controvertidos, polarizados o falsos, suponen una clara amenaza contra los derechos de diversos colectivos en peligro de exclusión social y que también participan del espacio online. Por ello, las desigualdades en el ciberespacio no han hecho más que crecer durante los últimos años, en multitud de formas, ya sea entre los que desarrollan las plataformas y consumen sus contenidos, entre los que las explotan en su beneficio y los que son explotados por ellas, entre los que se aprovechan de ellas para manipular y los que son manipulados o entre los países que se benefician de sus impuestos y los que no lo hacen, por citar algunas de ellas. El diseño de este tipo de plataformas que impulsa el capitalismo digital, pese a auto-posicionarse como neutro e igualitario, dista mucho de serlo y podemos encontrar multitud de casos en los que la manipulación informativa y/o la desinformación han campado a sus anchas por este tipo de plataformas, como en el reciente caso del COVID-19. No hay que olvidar que todas estas plataformas disponen de una serie de valores y características que fomentan los contenidos virales y la hipersegmentación de sus usuarios, desde una administración vertical, férreamente controlada por los propietarios de la misma (M. L. Gray \& Suri, 2019; Kenney \& Zysman, 2016; Morozov, 2013; Zuboff, 2019).

Dicho de otro modo, los valores impresos en su desarrollo tecnológico y sus modelos de negocio impulsan y favorecen la constante conectividad, comunicación y codificación de las interacciones del usuario con las plataformas. Dichas interacciones con estos entornos son digitalizadas para posteriormente ser convertidas en datos y metadatos que puedan ser almacenados, tratados y procesados con el fin de crear diversos servicios a partir de perfiles de usuarios, preferencias de uso, etc. Al mismo tiempo, esta centralidad en la cultura popular contemporánea que han ido adquiriendo las plataformas digitales durante los últimos años supone una ventaja considerable para la difusión de estas nuevas interfaces basadas en la voz humana que se han ido desgranando en el presente texto. Las IC aspiran a facilitar la interacción con un entorno cada vez más complejo y mediatizado, a través de una comunicación oral. Si como una suerte de 'lazarillos digitales' fueran, las IC aspiran a acompañarnos en nuestra cotidianeidad a través de diferentes dispositivos (ordenadores, teléfonos, altavoces, televisores, relojes o robots) y de diferentes rutinas diarias (consultando la previsión meteorológica, ayudándonos en nuestras compras online, planificando nuestro próximo viaje o pinchando nuestra canción favorita). Algo que en un entorno cada vez más complejo, mediatizado y repleto de diversas innovaciones tecnológicas que requieren de un conocimiento técnico medio por parte del usuario, puede verse a priori como un avance a la hora de reducir la complejidad en las interacciones con los diversos dispositivos.

Sin embargo, el desarrollo de las IC entraña diversas reproducciones de desigualdades que ya se producen en la sociedad actual. Un ejemplo concreto lo tenemos en materia de igualdad de género, ya que es cuando menos curioso observar cómo se emplean comúnmente nom- 
bres femeninos dotados de connotaciones serviciales en la cultura popular, para atribuir de género a unos artefactos que no tendrían por qué disponer del mismo (Bell, 2017; Phan, 2019). Aquí los algoritmos que traccionan estas IC deben adoptar formas materiales y lingüísticas que se encuentran ampliamente asentadas en la sociedad para facilitar su difusión, pese a que dichas interfaces presenten otras opciones con voz no femenina (Phan, 2017). En el ámbito lingüístico, también se observa que las IC ahondan en las desigualdades asociadas a la popularización de Internet y la Web a lo largo del planeta. Si bien el desarrollo de estas tecnologías y plataformas digitales que impulsan el capitalismo digital han contribuido de manera unívoca al desarrollo de la sociedad de la información por todo el planeta, también han conllevado una uniformidad cultural y colonización digital de muchas comunidades y culturas minoritarias que no se encuentran adecuadamente representadas en el espacio online. De este modo, podemos observar una situación de sobrerrepresentación del idioma británico y la cultura anglosajona en general, ya que es en este contexto donde se enmarca el origen de todas estas plataformas digitales. Asistimos, pues, a una infrarrepresentación de culturas y lenguajes en el espacio digital, debido a la falta de recursos, infraestructuras y contenidos digitales para otro tipo de lenguas y contexto minoritarios.

En este sentido, el desarrollo de las IC representa una nueva vuelta de tuerca en estas desigualdades promovidas por el capitalismo digital, ya que estas nuevas interfaces promueven también una uniformidad en el uso de los lenguajes mayoritarios en el planeta y menosprecian la riqueza lingüística que generan acentos, coloquialismos, expresiones, dialectos, matices y tonos diversos que escapan a la estandarización de los corpus por parte de las tecnologías que se impulsan alrededor de la PLN y la IA. Entrenar a asistentes virtuales como Siri en varios idiomas requiere mucho tiempo y dinero, ya que supone implementar varios algoritmos y realizar un entrenamiento específico para cada idioma, al igual que implementar diferentes acentos y reconocer palabras o términos que se empleen por comunidades más minoritarias (Bosker, 2013). Estas decisiones no obedecen simplemente a cuestiones puramente técnicas, sino que los modelos de negocio digitales que están detrás del desarrollo de estas tecnologías son los que guían la toma de decisiones en torno a diversos aspectos de las mismas. Aquí nos encontramos una vez más con criterios de rentabilidad de la $\mathrm{I}+\mathrm{D}$ cortoplacistas y de interés privado que diseñan e imprimen valores en el desarrollo tecnológico, alejados del bien común.
La falta de inclusión, equidad, reflexividad y diversidad cultural que la industria de la IA manifiesta poseer es un fiel reflejo de las particularidades del contexto donde se desarrollan innovaciones como las IC. Las plataformas digitales que impulsan esta nueva revolución en los interfaces promueven una idea de progreso que se asocia fácilmente con desigualdad y 'solucionismo tecnológico’ (Morozov, 2013). La lógica de este capitalismo digital basado en la explotación de los datos de sus usuarios configura unas relaciones de poder en las que los dueños de estas infraestructuras digitales dejan de lado a una gran parte de la sociedad, fuera de los beneficios que produce. Esto hace que se revele como una imperiosa necesidad, la de complementar e intervenir en el desarrollo de dichas innovaciones con otro tipo de intereses de colectivos y minorías que no están propiamente representados en el espacio cibernético. En este sentido, iniciativas de código abierto como la que impulsa Mozilla denominada 'Common Voice' muestran un camino alternativo (Mozilla, 2020).

\section{Conclusiones y futuras direcciones}

En el presente texto se ha reflejado cómo las plataformas digitales se han revelado durante los últimos años como los máximos exponentes del capitalismo digital y cómo han basado sus estrategias de negocio alrededor de la extracción de plusvalías digitales de sus usuarios. Esto ha sido posible por el desarrollo de servicios, tecnologías, protocolos, dispositivos e interfaces orientadas a la recolección, almacenamiento y tratamiento de datos que puedan derivar en el desarrollo de ventajas competitivas.

En este sentido, el surgimiento de las IC a través del desarrollo de la IA, el AA y el PNL responde a la lógica que promueve un capitalismo basado en datos y que guíalos desarrollos de $\mathrm{I}+\mathrm{D}$ de las plataformas digitales. Por ello, se advierte de las numerosas implicaciones socio-éticas que presentan el desarrollo de las IC, y de las nuevas problemáticas que pueden introducirse en la sociedad, de la mano de su difusión.

Al mismo tiempo, es necesario resaltar las limitaciones de este artículo, las cuales radican en la ausencia de material empírico y por ello se anima al desarrollo de futuras investigaciones que aborden las múltiples dimensiones de la innovación que se producen en el capitalismo digital, ya sea en contextos donde se desarrollan dichas invenciones o en donde son difundidas, para una mejor comprensión de las diversas variables que las caracterizan y modelan.

\section{Referencias}

Allen, J. F., Byron, D. K., Dzikovska, M., Ferguson, G., Galescu, L., \& Stent, A. (2001). Toward Conversational Human-Computer Interaction. AI Magazine, 22(4), 27-38.

Artificial Intelligence Center. (n.d.). Cognitive Assistant that Learns and Organizes. Extraído el 7 de Julio de 2020 desde http:// www.ai.sri.com/project/CALO.

Barbrook, R., \& Cameron, A. (1996). The Californian ideology. Science as Culture, 6(1), 44-72. ht t p s:// d o i o org/1 0 . 1080 / 09505439609526455.

Bell, K. (2017). Hey, Siri: How'd you and every other digital assistant get its name? Extraído el 7 de mayo de 2020 desde https:// mashable.com/2017/01/12/how-alexa-siri-got-names/?europe=true. 
Bosker, B. (2013). SIRI RISING: The Inside Story Of Siri’s Origins - And Why She Could Overshadow The iPhone. Extraído el 7 de Julio de 2020 desde http://www.huffingtonpost.com/2013/01/22/siri-do-engine-apple-iphone_n_2499165.html.

Botsman, R., \& Rogers, R. (2011). What's mine is yours. How collaborative consumption is changing the way we live. London: Harper Collins.

Dourish, P. (2016). Algorithms and their Others: Algorithmic Culture in Context. Big Data \& Society, (December), 1-11. https:// doi.org/10.1177/2053951716665128.

Geller, T. (2012). Talking to Machines. Communications of the ACM, 55, 14-16. https://doi.org/10.1145/2133806.2133812

Gillespie, T. (2010). The Politics of Platforms. New Media \& Society, 12(3), 347-364. https://doi.org/10.1002/9781118321607. $\operatorname{ch} 28$.

Gray, C. (2014). Big Data, Actionable Information, Scientific Knowledge and the Goal of Control. Teknokultura, 11(3), 529-554.

Gray, M. L., \& Suri, S. (2019). Ghost Work: How to Stop Silicon Valley from Building a New Global. Boston: Houghton Mifflin Harcourt.

Gutiérrez, R. T. (2016). El surgimiento de HTML5; un nuevo paradigma en los estándares Web. Revista Teknokultura, 13(1), 169-192. https://doi.org/10.5209/rev_TK.2016.v13.n1.52152.

Gutiérrez, R. T. (2018). Understanding the role of digital commons in the web; The making of HTML5. Telematics and Informatics, 35(5), 1438-1449. https://doi.org/10.1016/j.tele.2018.03.013.

Gutiérrez, R. T., \& Ezponda, J. E. (2019). Technodata and the Need of a Responsible Industry 4.0. In J. P. Z. Bonilla \& V. G.-P. Diaz (Eds.), Handbook of Research on Industrial Advancement in Scientific Knowledge (pp. 1-19). Hershey: IGI Global. https://doi.org/10.4018/978-1-5225-7152-0.

Guzman, A. L. (2017). Making AI safe for humans: A conversation with Siri. In R. W. Gehl \& M. Bakardjieva (Eds.), Socialbots and Their Friends: Digital Media and the Automation of Sociality (pp. 69-85). New York: Routledge. https://doi. org/10.4324/9781315637228.

Guzman, A. L. (2018). Voices in and of the machine: Source orientation toward mobile virtual assistants. Computers in Human Behavior, 90, 343-350. https://doi.org/10.1016/j.chb.2018.08.009.

Hallinan, B., \& Striphas, T. (2014). Recommended for you: The Netflix Prize and the production of algorithmic culture. New Media \& Society, 1461444814538646-. https://doi.org/10.1177/1461444814538646.

Kenney, M., \& Zysman, J. (2016). The Rise of the Platform Economy. Issues in Science and Technology, 32 (3), 61.

Knight, W. (2016a). Conversational Interfaces. Powerful speech technology from China's leading Internet company makes it much easier to use a smartphone. Extraído el 9 de Julio de 2020 desde https://www.technologyreview.com/s/600766/10breakthrough-technologies-2016-conversational-interfaces/.

Knight, W. (2016b). Tougher Turing Test Exposes Chatbots' Stupidity. Extraído el 7 de Julio de 2020 desde https://www. technologyreview.com/s/601897/tougher-turing-test-exposes-chatbots-stupidity/.

Lai, J. (2000). Conversational Interfaces. Communications of the ACM, 43(9), 24-27.

Morozov, E. (2013). To save everything, click here: The folly of technological solutionism. New York: Public Affairs.

Mozilla. (2020). Common Voice. Extraído el 7 de Julio de 2020 desde https://voice.mozilla.org/es/about.

O'Neill, C. (2017). Weapons of Math Destruction. How Big Data increases inequality and threatens democracy. London: Penguin Books.

O'Reilly, T. (2005). What Is Web 2.0? Design Patterns and Business Models for the Next Generation of Software. Extraído el 9 de Julio de 2020 desde http://oreilly.com/web2/archive/what-is-web-20.html.

Pasquale, F. (2015). The black box society: The secret algorithms that control money and information. London: Harvard University Press.

Phan, T. (2017). The Materiality of the Digital and the Gendered Voice of Siri. Transformations, 29(29), 23-33.

Phan, T. (2019). Amazon Echo and the Aesthetics of Whiteness. Catalyst: Feminism, Theory, Technoscience, 5(1), 1-38. https:// doi.org/10.28968/cftt.v5i1.29586.

Ritzer, G., \& Jurgenson, N. (2010). Production, Consumption, Prosumption: The nature of capitalism in the age of the digital "prosumer." Journal of Consumer Culture, 10(1), 13-36. https://doi.org/10.1177/1469540509354673.

Roush, W. (2010). The Story of Siri, from Birth at SRI to Acquisition by Apple-Virtual Personal Assistants Go Mobile. Extraído el 10 de Diciembre de 2019 desde https://xconomy.com/san-francisco/2010/06/14/the-story-of-siri-from-birth-at-sri-toacquisition-by-apple-virtual-personal-assistants-go-mobile/.

Scholz, T. (2012). Digital labor: The internet as playground and factory. New York: Routledge.

Scholz, T. (2017). Uberworked and Underpaid. How workers are disrupting the digital economy. Cambridge, UK: Polity Press.

Srnicek, N. (2017). Platform Capitalism. Cambridge, UK: Polity Press.

Stefano, V. De. (2016). The rise of the "Just-in-time workforce": On-demand work, crowdwork, and labor protection in the "gigeconomy." Comp. Lab. L. \& Pol'y J., 37(3), 471.

Sundararajan, A. (2016). The Sharing Economy. Cambridge, MA: The MIT Press.

Terranova, T. (2000). Free Labor: Producing Culture for the Digital Economy. Social Text, 18(2), 33-58. https://doi. org/10.1215/01642472-18-2_63-33.

Turner, F. (2006). From Counterculture to Cyberculture: Stewart Brand, the Whole Earth Network, and the Rise of Digital Utopianism. Chicago: University of Chicago Press.

van Dijck, J. (2013). The Culture of Connectivity: A Critical History of Social Media. New York: Oxford University Press.

van Dijck, J., Poell, T., \& Waal, M. de (2018). The platform society: Public values in a connective world. New York: Oxford University Press.

Vogelstein, F. (2013). Dogfight: How Apple and Google Went to War and Started a Revolution. Sarah Crichton Books.

Zuboff, S. (2019). The Age of Surveillance Capitalism: The Fight for a Human Future at the New Frontier of Power. London: Profile Books.

Zue, V. W., \& Glass, J. R. (2000). Conversational interfaces: advances and challenges. Proceedings of the IEEE, 88(8), 11661180. https://doi.org/10.1109/5.880078. 